\title{
Sleep disorders in patients with psoriatic arthritis and psoriasis
}

\author{
Magdalena Krajewska-Włodarczyk ${ }^{1}$, Agnieszka Owczarczyk-Saczonek², Waldemar Placek ${ }^{2}$ \\ ${ }^{1}$ Department of Internal Medicine, School of Medicine, Collegium Medicum, University of Warmia and Mazury, Poland \\ 2Department of Dermatology, Sexually Transmitted Diseases and Clinical Immunology, School of Medicine, Collegium Medicum, \\ University of Warmia and Mazury, Poland
}

\begin{abstract}
Objectives: To assess and measure occurrence of sleep disorders in patients with psoriatic arthritis (PsA) and psoriasis (Ps).

Material and methods: The study included 62 patients with psoriatic arthritis and 52 patients with psoriasis. The measurement of sleep quality was conducted using the Pittsburgh Sleep Quality Index (PSQI), the evaluation of fatigue by the fatigue subscale of the FACIT-F questionnaire and the patient's quality of life by the Health Assessment Questionnaire (HAQ). The psoriasis severity was determined using the Psoriasis Area Severity Index (PASI) and the activity of arthritis by the disease activity score of 28 joints (DAS 28). The Visual Analogue Scale (VAS) was used to assess the severity of pain.

Results: Poor sleep quality was found in $67.7 \%$ of PsA patients, $57.7 \%$ in Ps patients and $14.6 \%$ within the control group. Sleeping disorders in patients with PsA and Ps were related to worse quality of life and intense fatigue. Methotrexate treatment was not related to sleeping disorders, but an improvement in sleep quality was observed in both PsA and Ps patients who were treated with antiTNF- $\alpha$ antibodies ( $p<0.001$ and $p=0.032$ respectively). Following the use of the linear regression model, the following factors worsen the sleep quality in PsA: pain $\left(R^{2}=0.462, p<0.001\right)$, tender joint count $\left(R^{2}=0.434, p<0.001\right)$, C-reactive protein (CRP) concentration $\left(R^{2}=0.391, p<0.001\right)$, patient's age $\left(R^{2}=0.284, p=0.003\right)$ and duration of psoriasis $\left(R^{2}=0.166, p=0.006\right)$. In Ps patients the factors were: severity of skin lesions $\left(R^{2}=0.329, p<0.001\right)$, duration of psoriasis $\left(R^{2}=0.290\right.$, $p=0.004)$ and patient's age $\left(R^{2}=0.282, p=0.019\right)$.

Conclusions: Poor sleep quality in patients with PsA or Ps is a common symptom. Sleep disorders are more frequent in patients with PsA than in those with psoriasis.
\end{abstract}

Key words: psoriatic arthritis, pain, sleep disorders, fatigue.

\section{Introduction}

Psoriatic arthritis (PSA) is a chronic inflammatory disease of the joints, which belongs to the group of spondyloarthritis, associated with psoriasis (Ps). The clinical portrayal of PSA in addition to peripheral or axial joint involvement includes changes in the enthesitis and dactylitis as well as numerous non-articular manifestations. Both PSA and Ps, apart from the obvious effects on the physical condition of the patient, are associated with deterioration of social functioning and with psychological problems [1]. More and more often, apart from the assessment of the local skin condition including the effects of the disease and accompanying symptoms such as pruritus and pain, the psychological aspects in PsA and Ps patients are also evaluated. So far the impact of the disease on the quality of life $(\mathrm{Q} o \mathrm{~L})$ of patients suffering from PsA and Ps has been well described [2-4]. It seems that PsA and Ps can significantly affect the feeling of increased fatigue [5] and sleep disorders compared to the general population [6]. Long-term extensive deterioration of sleep quality not only lowers the quality of life, 
but also leads to the development of hypertension [7] cardiovascular complications [8] and metabolism disorders [9], which are significant effects of PSA and Ps. In addition, even short-term sleep deficits may lead to increased synthesis and the release of proinflammatory cytokines such as TNF- $\alpha, \mathrm{IL}-1 \beta, \mathrm{IL}-17$ and C-reactive protein (CRP) $[8,10,11]$. In the course of suffering from PsA and Ps, the regularity and severity of sleep disorders may be associated with inflammation, chronic pain and pruritus, decreased quality of life caused by the disease, emotional disorders such as anxiety and depressive reactions and adverse reactions to current medication [1].

The aim of the study was to assess sleep disorders and the relations of worsening quality of sleep to selected clinical parameters in investigated patients with psoriatic arthritis (PsA), psoriasis (Ps) and a control group.

\section{Material and methods}

All consecutive patients with psoriasis and with psoriatic arthritis who attended the Rheumatology and Dermatology Clinic and the Department of Rheumatology of the Municipal Hospital in Olsztyn and the Department of Dermatology, Sexually Transmitted Diseases and Clinical Immunology of the University of Warmia and Mazury in Olsztyn, Poland for a three-month period (March-May 2018) were enrolled in this study. The study included 62 patients (33 women, 29 men) with psoriatic arthritis and 52 patients ( 27 women, 25 men) with psoriasis alone. The control group consisted of 41 people (21 women, 20 men) without psoriasis or psoriatic arthritis. The age of the examined subjects was in the range of 18 to 60 years old. The diagnosis of psoriatic arthritis was established on the basis of CASPAR criteria [12].

Patients with hyperthyroidism and hypothyroidism, as well as with heart, pulmonary, liver or renal insufficiency and malignancies, were excluded from the study.

In the dermatological examination, the degree of psoriasis severity was determined using PASI (Psoriasis Area Severity Index) [13]. The activity of joint disease was assessed using the Disease Activity Score of 28 joints (DAS 28) [14] and the number of painful and swollen joints. The Visual Analogue Scale (VAS) was used to assess the pain level.

Measurement of sleep quality was conducted using the Pittsburgh Sleep Quality Index (PSQI) [15]. The scale consists of 19 items and assesses seven domains: subjective sleep quality, sleep latency, sleep duration, habitual sleep efficiency, sleep disturbances, use of sleeping medications and daytime dysfunctions. Scores in each component of sleep quality range from 0 to 3 and the global PSQI ranges from 0 to 21 . A total score of $\geq 6$ is considered as poor sleep quality.
The quality of life of patients was assessed using a health assessment questionnaire (HAQ) [16].

The evaluation of fatigue was conducted using a validated Polish version of the fatigue subscale of the FACIT-F questionnaire. In order to use the validated Polish version of the FACIT-F questionnaire in this study, written consent from the FACIT organization was obtained (issued on 30/01/2017). A result of 24 points or less was adopted in order to evaluate the significance of fatigue [17].

Inflammatory markers were measured with two standard laboratory parameters: erythrocyte sedimentation rate (ESR) assessed using BD Vacutainer Sedi-15 equipment (BD, Franklin Lakes, NJ, USA) and concentration of C-reactive protein (CRP) measured with a standard immunoturbidimetric method using a COBAS 6000 INTEGRA apparatus (Roche Diagnostics, Mannheim, Germany).

All patients gave written consent to participate in the examination. The study was conducted in accordance with the recommendations of the Helsinki Declaration and was approved by the Bioethics Committee of the Warmia and Mazury Chamber of Physicians (OIL 625/16/Bioet; 21.12.2016).

\section{Statistical analysis}

StatSoft STATISTICA, version 12.5 (StatSoft, Tulsa, OK, USA) was used for calculations. Obtained results were presented as the arithmetic mean and standard deviation. The Mann-Whitney U-test and the KruskalWallis test were used for comparative analysis between the groups. The presence of a relationship between quantitative features was tested using Pearson's correlation coefficient for parameters consistent with a normal distribution and Spearman's correlation coefficient in cases of non-compliance with a normal distribution. To evaluate the relationship between the studied data, a linear regression model was used. Variable models were selected stepwise using backwards elimination. The statistical level of significance was $p<0.05$.

\section{Results}

The study included a total of 155 people aged 18-60 years, including 62 patients with psoriatic arthritis (33 women, 29 men), 52 patients with psoriasis (27 women, 25 men) and 41 people without inflammatory skin or joint disease (21 women, 20 men). The studied groups did not significantly differ in age. In patients suffering from psoriasis greater severity of skin lesions was observed in comparison to those with PsA. Patients with PsA however had significantly higher values of 
inflammatory parameters when examined. There was no significant difference in the amount of time the patients suffered from psoriasis (Table I).

In both groups of PsA and Ps patients, the sleep quality was assessed by the PSQI questionnaire and deteriorated with the increasing duration of skin disease (respectively $r=0.391, p<0.05$ vs. $r=0.283, p<0.05$ ) and age (respectively $r=0.379, p<0.05$ vs. $r=0.345$, $p<0.05)$. Deterioration of sleep quality caused by aging was also observed in the control group $(r=0.307$, $p<0.05)$. Among patients with PsA, there was no significant correlation between the deterioration of sleep quality and the duration of arthritis. The severity of skin lesions in patients without arthritis had a significant effect on sleeping disorders $(r=0.48, p<0.05)$; this relationship was not observed in the group with PSA. Among patients with PsA and Ps, sleep quality deteriorated according to the severity of pain caused by skin lesions or joint involvement (respectively: $r=0.392$, $p<0.05$ vs. $r=0.314, p<0.05$ ). The worsening sleep quality in PsA patients was associated with an increase in the number of tender joints $(r=0.369, p<0.05)$, increased CRP $(r=0.276, p<0.05)$, but not with either the number of swollen joints or with an increase in ESR. No relationship was observed between CRP concentration or high ESR levels and deterioration of sleep quality in patients with Ps. Educating the participants did not affect the results of the PSQI questionnaire. Among patients with PsA and Ps and the control group, sleep disorders were more frequently observed in women than in men (respectively: $p=0.024, p=0.008, p=0.017$ ).
Sleep disorders observed in those suffering from either PsA or Ps were associated with a deterioration of their life quality as assessed by the HAQ questionnaire $(r=0.394, p<0.05$ vs. $r=0.313, p<0.05)$. Also, the intensity of fatigue as assessed with the fatigue subscale of the FACIT-F questionnaire in both groups was related to decreased quality of sleep (respectively: $r=-0.412$, $p<0.05$ vs. $r=-0.348, p<0.05)$.

Among the examined PSA and Ps patients who were treated with methotrexate, there was no significant reduction in sleep quality in either group, while better sleep quality was observed in patients with PsA treated with anti-TNF- $\alpha$ antibodies $(p<0.001)$ and patients with Ps $(p=0.032)$.

Within the entire group of patients, when compared to the control group, worse sleep quality was observed, according to all the tested PSQI domains with the exception of the use of sleeping medication. Bad quality of sleep was determined as a score of 6 or more points, obtained via the PSQI questionnaire; it was found that $67.7 \%$ of patients with PsA, $57.7 \%$ of patients with Ps and $14.6 \%$ of subjects in the control group suffered from bad quality of sleep (Table II). In addition, the PsA patient group when compared to patients with Ps had a significant reduction in sleep quality which was observed in all assessed PSQI domains as well as the duration and use of sleeping medication (Table II).

After applying the linear regression model, based on including within the primary model all the potential variables selected with the stepwise method of retrograde elimination, factors that had the greatest impact on

Table I. Age and clinical characteristic of the patients

\begin{tabular}{|c|c|c|c|c|}
\hline & $\operatorname{PsA}(n=62)$ & $\operatorname{Ps}(n=52)$ & Control $(n=41)$ & $p$-value \\
\hline Gender (F/M) & $33 / 29$ & $27 / 25$ & $21 / 20$ & NS \\
\hline Age (years) & $47.8 \pm 8.9$ & $42.4 \pm 12.4$ & $42.4 \pm 11.6$ & NS \\
\hline Ps duration (years) & $19.3 \pm 8.9$ & $16.7 \pm 12.3$ & - & NS \\
\hline PsA duration & $8.4 \pm 7.4$ & - & - & - \\
\hline DAS 28 & $3.42 \pm 0.6$ & - & - & - \\
\hline PASI & $7.3 \pm 6.1$ & $10.4 \pm 7.1$ & - & 0.034 \\
\hline ESR & $22.6 \pm 11.4$ & $14.3 \pm 8.8$ & - & 0.029 \\
\hline CRP $(\mathrm{mg} / \mathrm{dl})$ & $9.6 \pm 5.8$ & $3.8 \pm 2.5$ & - & 0.011 \\
\hline HAQ & $0.89 \pm 0.5$ & $0.78 \pm 0.7$ & - & NS \\
\hline FACIT-F & $27.7 \pm 8.7$ & $36.8 \pm 7.5^{1}$ & $41.0 \pm 7.2$ & $<0.001$ \\
\hline VAS & $34.8 \pm 14.6$ & $26.4 \pm 19.9$ & - & NS \\
\hline TJC & $2.8 \pm 1.4$ & - & - & - \\
\hline SJC & $1.5 \pm 0.6$ & - & - & - \\
\hline
\end{tabular}

Results are presented as mean values and standard deviations (SD). PS - psoriasis; PSA - psoriatic arthritis; NS - not significant; DAS disease activity score; PASI - psoriasis area severity index; ESR - erythrocyte sedimentation rate; CRP - C-reactive protein; HAQ - health assessment questionnaire; VAS - visual analog scale; TJC - tender joint count; SJC - swollen joint count; ${ }^{1} p=0.031$ psoriasis Vs. control) 
Table II. The comparison of Pittsburgh Sleep Quality Index between patients studied

\begin{tabular}{|lcccc|}
\hline & PsA $(n=62)$ & Ps $(n=52)$ & Control $(n=41)$ & $p$-value \\
\hline PSQI $\geq 6, n(\%)$ & $42(67.7)$ & $30(57.7)$ & $6(14,6)$ & $<0.001$ \\
\hline Global PSQI (0-21) & $9.32 \pm 5.02$ & $7.11 \pm 4.31^{1}$ & $4.05 \pm 2.91$ & $<0.001$ \\
\hline Subjective sleep quality (0-3) & $1.48 \pm 1.31$ & $1.31 \pm 1.22^{2}$ & $0.89 \pm 0.78$ & 0.002 \\
\hline Sleep latency (0-3) & $1.52 \pm 1.21$ & $1.37 \pm 1.19^{3}$ & $1.23 \pm 1.09$ & 0.042 \\
\hline Sleep duration (0-3) & $1.43 \pm 1.14$ & $1.39 \pm 1.16^{4}$ & $1.18 \pm 0.98$ & 0.024 \\
\hline Habitual sleep efficiency (0-3) & $2.12 \pm 1.41$ & $1.76 \pm 1.29$ & $1.03 \pm 0.76$ & $<0.001$ \\
\hline Sleep disturbances (0-3) & $1.72 \pm 1.24$ & $1.42 \pm 1.18$ & $0.95 \pm 0.48$ & $<0.001$ \\
\hline Use of sleeping medication (0-3) & $1.17 \pm 0.91$ & $0.97 \pm 0.65$ & $0.87 \pm 0.68$ & NS \\
\hline Daytime disfunction (0-3) & $1.85 \pm 0.93$ & $1.56 \pm 1.15$ & $0.76 \pm 0.43$ & $<0.001$ \\
\hline
\end{tabular}

Results are presented as mean values and standard deviations (SD). PS - psoriasis; PSA - psoriatic arthritis; NS - not significant; PSQI - Pittsburgh Sleep Quality Index; ${ }^{1} p=0.027$ Ps vs. PSA; ${ }^{2} p=0.038$ PS vs. PSA; ${ }^{3} p=0.042$ PS vs. PSA; ${ }^{4} p=0.087$ PS vs. PSA

Table III. The regression coefficients in modelling for sleep quality in psoriatic arthritis

\begin{tabular}{|ccccccc|}
\hline & VAS & TJC & CRP & Age & Ps duration & Adjusted $R^{2}$ \\
\hline PSA $(n=62)$ & 0.462 & 0.434 & 0.391 & 0.284 & 0.166 & 0.452 \\
& {$[0]$} & {$[0]$} & {$[0]$} & {$[0.003]$} & {$[0.006]$} & \\
\hline
\end{tabular}

The p-value is shown in brackets. PSA - psoriatic arthritis; TJC - tender joint count; CRP-C-reactive protein; Ps - psoriasis

Table IV. The regression coefficients in modelling for sleep quality in psoriasis

\begin{tabular}{|lcccc|}
\hline & PASI & Ps duration & Age & Adjusted $R^{2}$ \\
\hline Ps $(n=52)$ & 0.329 & 0.290 & 0.282 & 0.301 \\
& {$[0]$} & {$[0.004]$} & {$[0.019]$} & \\
\hline
\end{tabular}

The $p$-value is shown in brackets. Ps - psoriasis; PASI - psoriasis area severity index

worsening sleep quality in the examined patients were determined. Factors having an effect on the reduction of sleep quality in patients with PSA were: increased joint pain, number of aching joints, CRP, age and duration the patients suffered from psoriasis (Table III).

Within the group of psoriasis patients, the factors affecting the quality of sleep were: severity of skin lesions assessed by PASI, duration the patients suffered from psoriasis and age of patients (Table IV).

\section{Discussion}

Sleep disorders associated with psoriasis and psoriatic arthritis may be a serious problem, yet one barely taken seriously, in everyday clinical practice [18]. So far, there have been few studies on sleep disorders in PsA patients. In our study, significantly reduced sleep quality was found in $67.7 \%$ of patients with PsA. In the study conducted by Gezer et al. [19], sleep disorders in people with PsA were observed even more frequently, as they were observed in more than $85 \%$ of patients. In the course of ankylosing spondylitis (AS), another disease from the group of spondyloarthritis, similarly to our study, worsened quality of sleep was found in about $60 \%$ of patients $[20,21]$. Sleep disorders were observed in $57.7 \%$ of Ps patients. Similar results were reported by Melikoglu, [22] and Jensen et al. [23], where poor sleep quality was found in $60 \%$ and $54 \%$ of patients respectively. On the other hand, significantly worse sleep quality in patients suffering from Ps (more than 76\%) was observed by Henry et al. [24].

In our study, the deterioration in sleep quality of PsA and Ps patients compared to the control group was related to the following domains: subjective sleep quality, sleep latency, sleep duration, habitual sleep efficiency, sleep disturbances, and daytime dysfunction. No differences were observed between the studied patients and the control group in the amount of sleeping medication consumed.

Many reported factors may affect the quality of sleep in patients suffering from either PsA or Ps, such as pruritus, pain, depression and obstructive sleep apnea [6]. The sleep quality of the examined patients decreased gradually with the time patients suffered from psoriasis. No relationship between the duration of arthritis and the occurrence of sleep disorders was observed. Among the Ps patients, deterioration in the sleep quality was 
related to the severity of psoriatic lesions. The severity of skin lesions assessed by the PASI index related to the decreased sleep quality was also described in studies by Melikoglu [22] and Shutty et al. [25], while Gezer et al. [19] and Stinco et al. [26] did not observe this relationship. Significant deterioration in sleep quality in those with PsA compared to those with Ps was observed in all of the domains studied apart from sleep duration and use of sleeping medication. The number of painful but non-swollen joints affected the deterioration of sleep quality for the examined PsA patients. In our study, pain level was assessed using an analogue scale, and was found to be one of the main factors which worsened the sleep quality in patients suffering from PsA, as in the study conducted by Gezer et al. [19].

In contrast to that study, we did not observe a relationship between the deterioration of sleep quality and the activity of PsA disease assessed by DAS 28. Callis Duffin et al. [27] described the presence of arthritis as the most important factor impairing sleep in Ps patients. Although Strober et al. [28] did not find any relation between sleep disorders and pain, or pruritus, which can be one of the symptoms of Ps and PsA, in their study the coexistence of arthritis in patients with Ps was linked to patient's sleep quality deterioration. In the study conducted by Gezer et al. [19], the quality of sleep in PSA patients decreased with the increase of CRP and ESR, similarly to the study by Li et al. [21]. In our study, patients suffering with PsA had sleep disorders linked to an increase in CRP, but not ESR. No significant relationship between the increase in CRP concentration or OB levels and deterioration of sleep quality in Ps patients was observed.

Deterioration in the quality of life may affect sleep similarly to fatigue, which can be an obvious consequence of suffering from sleep disorders long term. Like in other studies, in our study, as expected, sleep disorders among patients suffering from PsA and Ps were linked with deterioration of life quality assessed using the HAQ questionnaire and with the increase of fatigue assessed by the fatigue subscale of the FACIT-F questionnaire in both groups of patients $[19,29]$.

No link was observed between sleep disorders in patients under methotrexate treatment, similar to the Gezer et al. study [19], while in the group of patients treated with anti-TNF- $\alpha$, the quality of sleep was significantly better than among other patients. An improvement in sleep quality among PsA and Ps patients during anti-TNF- $\alpha$ therapy was described by Strober et al. [28] and Wu et al. [30].

\section{Conclusions}

Poor sleep quality in patients suffering from PsA or $P S$ is a frequent symptom which is overlooked during routine medical examinations. The deterioration in the quality of sleep is related to the deterioration of the patient's life quality and the severity of experienced fatigue. In our study, sleep disorders were significantly more frequent in patients with arthritis. The main factors affecting the occurrence of sleep disorders in PSA patients were: the severity of pain assessed by the VAS scale, number of painful joints, increase in CRP, age of patients and time the patient suffered from psoriasis. In the group of patients suffering from Ps alone, in addition to the age of patients and the duration of suffering from psoriasis, the deterioration in sleep quality was related to the severity of skin disease assessed using the PASI index. Worsening of sleep quality in patients with PSA and Ps may be triggered by many factors, and it also seems to lead to other disorders. Due to the multidimensional nature of this problem, treatment of sleep disorders in PsA and Ps patients, aside from sleeping pills, should also include psychotherapy and effective treatment of the underlying disease.

The authors declare no conflict of interest.

\section{References}

1. Mease PJ, Menter MA. Quality-of-life issues in psoriasis and psoriatic arthritis: outcome measures and therapies from a dermatological perspective. J Am Acad Dermatol 2006; 54: 685-704.

2. Gudu T, Gossec L. Quality of life in psoriatic arthritis. Expert Rev Clin Immunol 2018; 14: 405-417.

3. Husni ME, Merola JF, Davin S. The psychosocial burden of psoriatic arthritis. Semin Arthritis Rheum 2017; 47: 351-360.

4. Tezel N, Yilmaz Tasdelen $\mathrm{O}$, Bodur $\mathrm{H}$, et al. Is the health-related quality of life and functional status of patients with psoriatic arthritis worse than that of patients with psoriasis alone? Int J Rheum Dis 2015; 18: 63-69.

5. Krajewska-Włodarczyk M, Owczarczyk-Saczonek A, Placek W. Fatigue - an underestimated symptom in psoriatic arthritis. Reumatologia 2017; 55: 125-130.

6. Gowda S, Goldblum OM, McCall WV, et al. Factors affecting sleep quality in patients with psoriasis. J Am Acad Dermatol 2010; 63: 114-123.

7. Thomas SJ, Calhoun D. Sleep, insomnia, and hypertension: current findings and future directions. J Am Soc Hypertens 2017; 11: 122-129.

8. van Leeuwen WMA, Lehto $M$, Karisola P, et al. Sleep restriction increases the risk of developing cardiovascular diseases by augmenting proinflammatory responses through IL-17 and CRP. PLoS One 2009; 4: e4589.

9. Van Cauter E, Spiegel K, Tasali E, et al. Metabolic consequences of sleep and sleep loss. Sleep Med 2008; 9: 23-28.

10. Vgontzas AN, Zoumakis E, Bixler EO, et al. Adverse effects of modest sleep restriction on sleepiness, performance, and 
inflammatory cytokines. J Clin Endocrinol Metab 2004; 89: 2119-2126.

11. Irwin MR, Olmstead R, Carroll JE. Sleep Disturbance, Sleep Duration, and Inflammation: A Systematic Review and Meta-Analysis of Cohort Studies and Experimental Sleep Deprivation. Biol Psychiatry 2016; 80: 40-52.

12. Taylor W, Gladman D, Helliwell P, et al. Classification criteria for psoriatic arthritis: development of new criteria from a large international study. Arthritis Rheum 2006; 54: 2665 2673.

13. Mrowietz U, Kragballe K, Reich K, et al. Definition of treatment goals for moderate to severe psoriasis: a European consensus. Arch Dermatol Res 2011; 301: 1-10.

14. Praevoo ML, van't Hof MA, Kuper HH, et al. Modified disease activity scores that include twenty-eight-joint counts. Arthritis Rheum 1995; 38: 44-48.

15. Buysse DJ, Reynolds CF 3rd, Monk TH, et al. The Pittsburgh Sleep Quality Index: a new instrument for psychiatric practice and research. Psychiatry Res 1989; 28: 193-213.

16. Bruce B, Fries JF. The Stanford health assessment question naire (HAQ): a review of its history, issues, progress, and documentation. J Rheumatol 2003; 30: 167-178.

17. www.facit.org/FACITOrg/Questionnaires (access on 30.01.2017).

18. Henry AL, Kyle SD, Bhandari S, et al. Measurement, classification and evaluation of sleep disturbance in psoriasis: a systematic review. PLoS One 2016; 11: e0157843.

19. Gezer O, Batmaz I, Sariyildiz M A, et al. Sleep quality in patients with psoriatic arthritis. Int J Rheum Dis 2017; 20: 1212 1218.

20. Nie A, Wang C, Song Y, et al. Prevalence and factors associated with disturbed sleep in outpatients with ankylosing spondylitis. Clin Rheumatol 2018; 37: 2161-2168.
21. Li Y, Zhang S, Zhu J, et al. Sleep disturbances are associated with increased pain, disease activity, depression, and anxiety in ankylosing spondylitis: a case-control study. Arthritis Res Ther 2012; 14: R215.

22. Melikoglu M. Sleep Quality and its Association with Disease Severity in Psoriasis. Eurasian J Med 2017; 49: 124-127.

23. Jensen P, Zachariae C, Skov L, et al. Sleep disturbance in psoriasis - a case-controlled study. $\mathrm{Br} J$ Dermatol 2018. doi: 10.1111/bjd.16702 [Epub ahead of print].

24. Henry AL, Kyle SD, Chisholm A, et al. A cross-sectional survey of the nature and correlates of sleep disturbance in people with psoriasis. Br J Dermatol 2017; 177: 1052-1059.

25. Shutty BG, West C, Huanq KE, et al. Sleep disturbance in psoriasis. Dermatol Online J 2013; 19: 1.

26. Stinco G, Trevisan G, Piccirillo F, et al. Psoriasis vulgaris does not adversely influence the quality of sleep. $G$ Ital Dermatol Venereol 2013; 148: 655-669.

27. Callis Duffin K, Wong B, Horn EJ, et al. Psoriatic arthritis is a strong predictor of sleep interference in patients with psoriasis. J Am Acad Dermatol 2009; 60: 604-608.

28. Strober BE, Sobell JM, Duffin KC, et al. Sleep quality and other patient-reported outcomes improve after patients with psoriasis with suboptimal response to other systemic therapies are switched to adalimumab: results from PROGRESS, an openlabel Phase IIIB trial. Br J Dermatol 2012; 167: 1374-1381.

29. Carneiro C, Chaves M, Verardino G, et al. Fatigue in psoriasis with arthritis. Skinmed 2011; 9: 34-37.

30. Wu C-Y, Chang Y-T, Juan C-K, et al. Depression and insomnia in patients with psoriasis and psoriatic arthritis taking tumor necrosis factor antagonists. Medicine 2016; 95: e3816. 